\title{
Performance of synthetic antiferromagnetic racetrack memory: domain wall versus skyrmion
}

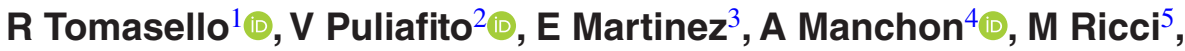 \\ M Carpentieri ${ }^{6}$ and G Finocchio ${ }^{7,8}$ (i) \\ ${ }^{1}$ Department of Engineering, Polo Scientifico e Didattico di Terni, University of Perugia, strada di \\ Pentima Bassa, I-05100 Terni, Italy \\ 2 Department of Engineering, University of Messina, C.da di Dio, I-98166, Messina, Italy \\ 3 Department of Fisica Aplicada, Universidad de Salamanca, Plaza de los Caidos s/n, E-38008, \\ Salamanca, Spain \\ ${ }^{4}$ Physical Science and Engineering Division (PSE), King Abdullah University of Science and Technology \\ (KAUST), Thuwal 23955-6900, Saudi Arabia \\ 5 Department of Computer Science, Modelling, Electronics and System Science, University of Calabria, \\ via P. Bucci, I-87036 Rende (CS), Italy \\ 6 Department of Electrical and Information Engineering, Politecnico di Bari, via E. Orabona 4, I-70125 \\ Bari, Italy \\ ${ }^{7}$ Department of Mathematical and Computer Sciences, Physical Sciences and Earth Sciences, University \\ of Messina, V.le F. D'alcontres, 31, I-98166, Messina, Italy
}

E-mail: gfinocchio@unime.it

Received 20 April 2017, revised 6 June 2017

Accepted for publication 20 June 2017

Published 18 July 2017

\begin{abstract}
A storage scheme based on racetrack memory, where the information can be coded in a domain or a skyrmion, seems to be an alternative to conventional hard disk drive for high density storage. Here, we perform a full micromagnetic study of the performance of synthetic antiferromagnetic (SAF) racetrack memory in terms of velocity and sensitivity to defects by using experimental parameters. We find that, to stabilize a SAF skyrmion, the DzyaloshinskiiMoriya interaction in the top and the bottom ferromagnet should have an opposite sign. The velocity of SAF skyrmions and SAF Néel domain walls are of the same order and can reach values larger than $1200 \mathrm{~m} \mathrm{~s}^{-1}$ if a spin-orbit torque from the spin-Hall effect with opposite sign is applied to both ferromagnets. The presence of disordered anisotropy in the form of randomly distributed grains introduces a threshold current for both SAF skyrmions and SAF domain walls motions.
\end{abstract}

Keywords: micromagnetism, spintronics, racetrack memory, domain wall, skyrmion, Dzyaloshinskii-Moriya interaction, spin-Hall effect

S Supplementary material for this article is available online

(Some figures may appear in colour only in the online journal)

\footnotetext{
${ }^{8}$ Author to whom any correspondence should be addressed.
} 


\section{Introduction}

The advent of a scenario where 'cloud computing' and 'internet of things' are merged together is pushing research efforts to find a technology that can go beyond the complementary metal-oxide semiconductor (CMOS), especially in terms of storage and computing [1]. Spintronics, with its subfields, is a promising candidate for that, owed to the possibility to use the degree of freedom of the spin angular momentum in addition to the charge of the electrons. Concerning the storage, two different categories of devices are promising: spin-transfertorque-MRAMs (STT-MRAMs) [1-5] and racetrack memories [6-10]. While STT-MRAMs (already on the market) can serve as 'universal memory' [1] (low writing energy, high read speed, and ideally infinite endurance) [2, 5], racetrack memories can be used as worthy alternative to hard disk drives in storage memory, with the advantage that no mechanical parts are necessary [9].

Since the first domain wall based racetrack memory presented in [6], performance improvements have been achieved by using materials with perpendicular anisotropy [11, 12], spin-orbit interactions, such as spin-Hall effect (SHE) $[13,14]$ and interfacial Dzyaloshinskii-Moriya interaction (IDMI)) $[7,8,15,16]$, and interlayer exchange coupling (IEC) [17, 18]. All previous ingredients gave rise to a racetrack memory where the perpendicular anisotropy and the IEC, together with the IDMI, stabilize DWs in synthetic antiferromagnets (SAFs) while SHE drives the DW motion at a velocity near 800 $\mathrm{m} \mathrm{s}^{-1}$ [18].

Recently, an alternative scheme of racetrack memory based on skyrmions has been proposed [19-23]. Skyrmions are topologically protected magnetic configurations that can be used for different applications [24-28]. In particular, in racetrack memories they can directly code the information, i.e. the presence/absence of the skyrmion represents the bit ' 1 '/'0' [19]. Skyrmion motion in ultrathin ferromagnetic materials has been demonstrated experimentally in extended $\mathrm{Ta} / \mathrm{CoFeB} /$ $\mathrm{TaO}$ multilayers [29], and in $\mathrm{Pt} / \mathrm{CoFeB} / \mathrm{MgO}$ [30], where a skyrmion velocity near $120 \mathrm{~m} \mathrm{~s}^{-1}$ has been measured. The main technological challenge to be overcome is the experimental control of a single skyrmion nucleation by an electrical current and its electrical detection [31, 32]. On the other hand, the main fundamental limitations of the skyrmion based racetrack memories are (i) the skyrmion Hall effect (two velocity components, parallel and perpendicular to the electrical current direction) [22, 33, 34] (ii) a transient breathing mode [22] and (iii) velocities below the ones of DWs for a fixed set of physical and geometrical parameters [19]. Lately, it has been shown that these issues could be solved by using a SAF skyrmion [35], opening a path for a more competitive skyrmion based racetrack memory. In that work [35], the tunnelling interlayer coupling was used to stabilize the SAF skyrmion [36, 37].

Here, we consider the same experimental framework by Yang et al [18] where the IEC is mediated by itinerant electrons via Ruderman-Kittel-Kasuya-Yosida (RKKY) field [38], resulting in an antiferromagnetic alignment of the two ferromagnets (other forms of IEC exist, such as Orange-Peel coupling, tunnelling etc). Firstly, we benchmark our computations with the experimental data on DWs, and then we predict the performance of SAF skyrmion based racetrack by considering realistic parameters, including edge roughness and bulk defects in the form of disordered grains with random uniaxial perpendicular anisotropy. Our key finding is that skyrmions in a SAF racetrack have velocities approaching the ones of DWs. We also show that a skyrmion based SAF racetrack is insensitive to edge roughness (an unavoidable type of defects in narrow nanotracks). On the other hand, the presence of a disordered anisotropy, considering different size of the grains, introduces a pinning of both SAF DW and SAF skyrmion, which is stronger when the average size of the grains is larger than the skyrmion diameter. In particular, this result shows that, when the grain size (GS) is larger than the skyrmion diameter, the pinning of the skyrmion originates only at the interface between two adjacent grains.

\section{Micromagnetic model}

We perform micromagnetic simulations of multilayered nanowires similar to the ones proposed in [18]. They are composed of a $3 \mathrm{~nm}$ thick Platinum heavy metal (HM) (lower $\mathrm{HM}$ ) with on top two perpendicular CoNi ferromagnetic layer (FMs) separated by a thin Ruthenium (Ru), layer designed to provide an antiferromagnetic exchange coupling [38] (see figure 1(a)), and a second HM on top of the whole stack (upper HM).

The nanowire is $100 \mathrm{~nm}$ wide and $1000 \mathrm{~nm}$ long and the thickness of both ferromagnets and Ru layer is $0.8 \mathrm{~nm}$. The physical parameters of CoNi layers taken from [17, 18], and equal for both ferromagnets, are: saturation magnetization $M_{\mathrm{s}}=600 \mathrm{kA} \mathrm{m}^{-1}$, exchange constant $A=20 \mathrm{pJ} \mathrm{m}^{-1}$, uniaxial perpendicular anisotropy constant $k_{\mathrm{u}}=0.6 \mathrm{MJ} \mathrm{m}^{-3}$, and damping parameter $\alpha_{G}=0.1$. The IEC constant $A^{\mathrm{ex}}$ is fixed to $-5.0 \times 10^{-4} \mathrm{~J} \mathrm{~m}^{-2}[17,18]$. We use a discretization cell of $4 \times 4 \times 0.8 \mathrm{~nm}^{3}$ (the results are robust against the discretization cell size, see appendix C), and introduce a Cartesian coordinate system with the $x$-, $y$ - and $z$-axes lying along the length, the width and the thickness of the wire, respectively (see figure 1(a)). The numerical study is carried out by means of a self-implemented micromagnetic solver (it includes the SHE, IDMI and IEC) and post-processing tools [39-41].

The total micromagnetic energy density of the system under investigation is (the superscripts $L$ and $U$ refer to lower and upper FMs):

$$
\begin{gathered}
\varepsilon_{\text {tot }}^{\mathrm{L}, \mathrm{U}}=A(\nabla \cdot \mathbf{m})^{2}+\varepsilon^{\mathrm{ex}} \\
+D^{\mathrm{L}, \mathrm{U}}\left[m_{z}^{\mathrm{L}, \mathrm{U}} \nabla \cdot \mathbf{m}^{\mathrm{L}, \mathrm{U}}-\left(\mathbf{m}^{\mathrm{L}, \mathrm{U}} \cdot \nabla\right) m_{z}^{\mathrm{L}, \mathrm{U}}\right] \\
-k_{u}\left(\mathbf{m}^{\mathrm{L}, \mathrm{U}} \cdot \hat{z}\right)^{2}-\frac{1}{2} \mu_{0} M_{\mathrm{s}} \mathbf{m}^{\mathrm{L}, \mathrm{U}} \cdot \mathbf{H}_{m}^{\mathrm{L}, \mathrm{U}}
\end{gathered}
$$

where $m_{x}, m_{y}$ and $m_{z}$ are the $x$-, $y$-, and $z$-components of the normalized magnetization $\mathbf{m}$, respectively. The interlayer exchange energy is given by $\varepsilon^{\mathbf{e x}}=-\frac{A^{\mathbf{e x}}}{t_{\mathbf{R U}}}\left(\mathbf{m}^{\mathbf{L}} \cdot \mathbf{m}^{\mathrm{U}}\right)$ (same contribution for both FMs), where $t_{\mathrm{RU}}$ is the thickness of the Ru layer [18]. $D$ is the parameter taking into account the intensity of the IDMI. More specifically, $D^{\mathrm{L}}\left(D^{\mathrm{U}}\right)$ refers to the 
lower (upper) FM. According to our definition, $D^{\mathrm{L}}$ and $D^{\mathrm{U}}$ are materials properties (they are not related to the multilayer geometry), i.e. in the specific case where both lower (HM/ FM) and upper (FM/HM) interfaces are the same, one would have $D^{\mathrm{L}}=D^{\mathrm{U}}$.

The Pt/FM interface produces the lower IDMI that we have swept from $0 \mathrm{~mJ} \mathrm{~m} \mathrm{~m}^{-2}$ to $4.0 \mathrm{~mJ} \mathrm{~m}^{-2}$, while the upper IDMI derives mainly from an upper HM. Notice that we disregard IDMI at $\mathrm{Ru}$ interfaces due to its small spin-orbit coupling [42]. $\hat{z}$ is the unit vector along the $z$-direction, $\mu_{0}$ is the vacuum permeability. $\mathbf{H}_{\mathrm{m}}$ is the magnetostatic dipolar field, which is computed by considering both ferromagnetic layers. The boundary conditions related to the IDMI are $\frac{\mathbf{d} \mathbf{m}^{\mathbf{L}, \mathbf{U}}}{\mathbf{d} n^{\mathrm{L}, U}}=\frac{1}{\xi^{\mathrm{L}, U}}\left(\hat{z} \times \mathbf{n}^{\mathbf{L}, \mathbf{U}}\right) \times \mathbf{m}^{\mathrm{L}, \mathrm{U}}[22,43,44]$, where $\mathbf{n}$ is the unit vector normal to the surface and $\xi^{\mathrm{L}, \mathrm{U}}=\frac{2 A}{D^{\mathrm{L}, \mathrm{U}}}$ is a characteristic length.

In the rest of the paper, the names DW and skyrmion refer to SAF configuration unless otherwise specified. Moreover, we wish to underline that, when considering the dynamical analysis, the presence of two HMs gives rise to a lower and upper SHE when an electric current is passed through them (the electrical current here denotes a flow of electrons). The spin-orbit torque due to the SHE is considered as a Slonczewski spin-transfer torque term for upper and lower FMs $\tau_{\mathbf{S H E}}^{\mathbf{L}, \mathbf{U}}=-\frac{g \mu_{\mathrm{B}} \theta_{\mathbf{S H}}^{\mathbf{L}, \mathbf{U}}}{2 \gamma_{0} e M_{\mathbf{S}}^{2} t_{\mathrm{FM}}^{\mathbf{L}, \mathbf{U}}} \mathbf{m}^{\mathbf{L}, \mathbf{U}} \times \mathbf{m}^{\mathbf{L}, \mathbf{U}}\left(\hat{z} \times \mathbf{j}_{\mathbf{H M}}^{\mathrm{L}, \mathrm{U}}\right)$, where $g$ is the Landè factor, $\mu_{\mathrm{B}}$ is the Bohr Magneton, $\gamma_{0}$ is the gyromagnetic ratio, $e$ is the electron charge, $t_{\mathrm{FM}}^{\mathrm{L}, \mathrm{U}}$ is the thickness of the lower/upper FM, $\hat{z}$ is the unit vector of the outof-plane direction and $\mathbf{j}_{\mathbf{H M}}^{\mathrm{L}, \mathrm{U}}$ is the in-plane current injected via the lower/upper HM. $\theta_{\mathrm{SH}}^{\mathrm{L}, \mathrm{U}}$ are the two spin-Hall angles for the lower and upper FMs, respectively. As for $D^{\mathrm{L}}$ and $D^{\mathrm{U}}$, according to our definition, the two spin-Hall angles are only linked to material properties.

\section{Results and discussion}

\subsection{Stability phase diagrams}

We firstly evaluate the stability phase diagram for both DW and skyrmion magnetic states as a function of the IDMI in the lower FM $\left(D^{\mathrm{L}}\right)$ and the IDMI ratio $\left(-D^{\mathrm{U}} / D^{\mathrm{L}}\right)$ with the aim to find a region where both states are stable.

Figure 1(b) shows these results, where we considered, as the initial state, a left-handed Néel DW for DW and Néel (hedgehog-like) skyrmion for the skyrmion. We display the region $-D^{\mathrm{U}} / D^{\mathrm{L}}>0$ being the only region of the phase diagram where skyrmion can be stabilized. This is the first important qualitative result of our study, i.e. the IDMI of the two HMs at the two HM/FM and FM/HM interfaces has to be opposite and then the upper and lower HMs should be different (see appendix A). In our case, because the lower HM is $\mathrm{Pt}$, the upper HM should be, for instance, W.

The phase diagram for the DW shows that a stable Néel DW (see snapshot figure 1(c)) is achieved for the whole range of parameters analyzed. For the skyrmion, three different regions
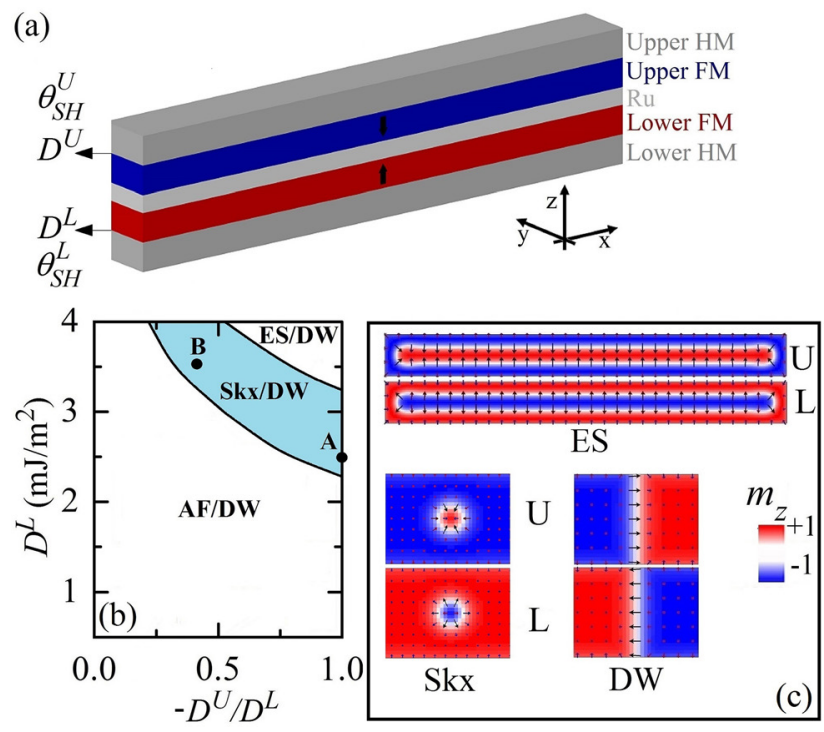

Figure 1. (a) Sketch of the SAF multilayer under investigation, where the ferromagnets (FM), separated by a Ru layer, are sandwiched between different heavy metals (HM). (b) Stability phase diagram for DW and skyrmion (the initial state is a lefthanded Néel DW for DW and Néel skyrmion for the skyrmion) of the magnetization state as a function of the lower IDMI parameter $D^{\mathrm{L}}$ and the IDMI ratio. The acronyms AF, DW, Skx and ES mean antiferromagnetic state, Néel domain wall, skyrmion and extended skyrmion, respectively. A and B refer to the working points considered for the study of DW and skyrmion motion. (c) Snapshots representing examples of the spatial distribution of the magnetization for DW (bottom right), Skx (bottom left), and ES (top). A color scale, linked to the $z$-component of the magnetization, is also indicated.

can be identified. The first one is characterized by the uniform antiferromagnetic state (AF), where the IDMI is not large enough to stabilize the skyrmion. The second region involves hedgehog-like or Néel skyrmion (Skx), which is achieved when $D^{\mathrm{L}}$ is large enough $\left(D^{\mathrm{L}} \geq 2.3 \mathrm{~mJ} \mathrm{~m}^{-2}\right)$. This threshold IDMI for the skyrmions stabilization is the first important difference with respect to the DW. The skyrmion core in the lower FM is oriented in the negative $z$-direction, with its DW spins pointing outward and the surrounded magnetization is along the positive $z$-direction. The upper skyrmion has an opposite orientation of the spins (core along the positive $z$-axis with inward DW spins at the transition region) and the magnetization pointing downward $(-z$-axis) (see snapshot in figure $1(\mathrm{c})$ ). At $D^{\mathrm{L}}=4.0 \mathrm{~mJ} \mathrm{~m} \mathrm{~m}^{-2}$, the minimum IDMI ratio $\left(-D^{\mathrm{U}} / D^{\mathrm{L}}\right)$ to stabilize skyrmions is $0.2 \mathrm{~mJ} \mathrm{~m}^{-2}$. In a previous paper [35], the skyrmion was stabilized without an upper HM $\left(D^{\mathrm{L}}\right.$ was set equal to $3.5 \mathrm{~mJ} \mathrm{~m}^{-2}$ ) because of different physical parameters.

The third region, obtained at very high $D^{\mathrm{L}}\left(>3.2 \mathrm{~mJ} \mathrm{~m}^{-2}\right)$, shows the stabilization of an extended skyrmion (ES), which is widened over the whole length of the strip. This state is linked to the bimeron instability (see snapshot of figure 1(c)), as well explained in [45], and its size is related to the confining potential given by both the magnetostatic energy and the IDMI boundary conditions.

In summary, the comparison between the two diagrams leads to the following main results: 
- A skyrmion is obtained only in a narrow range of $D^{\mathrm{L}}>0$ and positive IDMI ratio $\left(-D^{\mathrm{U}} / D^{\mathrm{L}}>0\right)$, while $\mathrm{DW}$ is stable for all the values. The stability of a skyrmion is also achieved for $D^{\mathrm{L}}<0$ and $-D^{\mathrm{U}} / D^{\mathrm{L}}>0$ (not shown) as can be understood from symmetry arguments.

- A region where both skyrmions and DWs are stable exists (cyan region in figure 1(b)), enabling their comparison by using the same set of parameters. In particular, for the dynamical analyses, we will focus on two working points 'A' $\left(D^{\mathrm{L}}=2.5 \mathrm{~mJ} \mathrm{~m}^{-2},-D^{\mathrm{U}} / D^{\mathrm{L}}=1.0\right)$ and 'B' $\left(D^{\mathrm{L}}=3.5 \mathrm{~mJ} \mathrm{~m}^{-2},-D^{\mathrm{U}} / D^{\mathrm{L}}=0.4\right)$ as indicated directly in figure 1(b);

- In those regions, the presence of either skyrmions or DWs depends on the nucleation process.

\subsection{SAF DW motion}

The first step of the dynamical study is the analysis of a SAF racetrack similar to the one proposed in [18], where the lower ferromagnet is coupled to a Pt HM, and no upper HM is considered. We use state-of-the-art values of $D^{\mathrm{L}}=2.5 \mathrm{~mJ} \mathrm{~m}^{-2}$ [46-48] and consider three different IDMI ratios $-D^{\mathrm{U}} / D^{\mathrm{L}}$ : $0.0,0.4$, and 1.0 (in [18], the authors claim that there might be an upper IDMI originated from the lower HM/FM interface and/or the Ru layer). We also take into account the effect of the edge roughness (two different patterns for the lower and upper FMs) as computed by the algorithm developed in [49]. In detail, the edge roughness is obtained by randomly removing regions from the strip edges with a uniform probability distribution characterized by the typical roughness size $D_{G}$. In our study, we consider a $D_{G}=12 \mathrm{~nm}$ [50] averaging the results of the velocity over five different random patterns. The electrical current $j_{\mathrm{HM}}$ is injected along the $x$-axis via the lower HM in order to originate the SHE only for the lower FM, with $\theta_{S H}^{L}=0.12$ [51].

Figure 2 summarizes the results of this study, where the curve with blue stars in figure 2(a) (experimental data from [18]), has been chosen as reference. The lower and upper DWs move tied along the $x$-direction because of the IEC (see supplemental movies 1 and 2 for $j_{\mathrm{HM}}=1.50$ and $3.25 \times 10^{8} \mathrm{Acm}^{-2}$, respectively (stacks.iop.org/JPhysD/50/325302/mmedia)). The velocity-current relation ( $v$ indicates the velocity along the $x$-axis) is independent of the IDMI ratio, such that the three numerical curves are almost overlapped. The pinning due to the edge roughness (see supplemental movie 3 for $j_{\mathrm{HM}}=0.50 \times 10^{8} \mathrm{~A} \mathrm{~cm}^{-2}$ ) gives rise to a threshold current to move the DW $\left(\left|j_{\mathrm{HM}}\right|>0.75 \times 10^{8} \mathrm{~A} \mathrm{~cm}^{-2}\right)$. As can be observed in the previous movie, the DW motion is characterized by a spatial tilting of its profile normal. The tilting angle of the DW spatial profile reverses symmetrically with the sign of the current (see also for comparison figure 1(b) in [18]), and it is robust against the edge roughness (compare supplemental movies 1 and 4, the latter obtained for a perfect SAF and $j_{\mathrm{HM}}=-1.50 \times 10^{8} \mathrm{~A} \mathrm{~cm}^{-2}$ ). Our simulations point out that the DW tilting is essentially due to the IDMI boundary conditions [52]. In fact, when the IDMI boundary conditions are neglected and regular exchange boundary conditions are used,

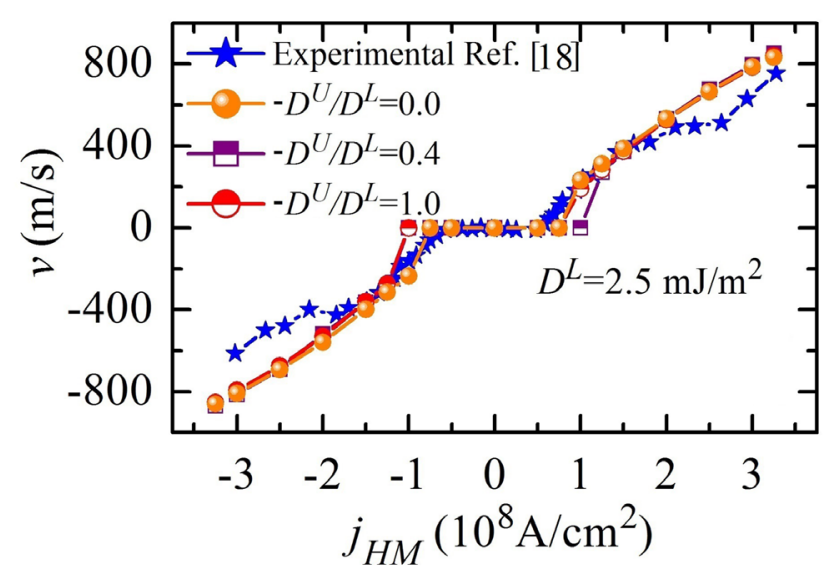

Figure 2. A comparison of the numerical velocity-current relation with the experimental one (blue stars in [18]). The computations concern a fixed $D^{\mathrm{L}}=2.5 \mathrm{~mJ} \mathrm{~m}^{-2}$, and three different IDMI ratios, as indicated in the legend. The edge roughness is taken into account for both ferromagnetic layers by means of the algorithm developed in [49] by using $D_{g}=12 \mathrm{~nm}$. The results are averaged over five different edge roughness distributions.

the DW profile normal keeps straight pointing along the $x$-axis (see supplemental movie 5 for a perfect SAF without IDMI boundary conditions and $j_{\mathrm{HM}}=-1.50 \times 10^{8} \mathrm{~A} \mathrm{~cm}^{-2}$ ). In addition, as the IDMI ratio increases, the tilting angle reduces for a fixed value of the current (see supplemental movie 6 for a perfect SAF, $j_{\mathrm{HM}}=-1.50 \times 10^{8} \mathrm{~A} \mathrm{~cm}^{-2}$ and $\left.-D^{\mathrm{U}} / D^{\mathrm{L}}=1.0\right)$. In other words, the larger is the current, the larger is the tilting. From this analysis to benchmark our model, we can conclude that the numerical outcomes are in good agreement with the experimental findings and the numerical model can be used for the predictions of dynamical properties of DW and skyrmion in similar systems.

In the rest of the paper, we focus on the parameters related to the working points ' $A$ ' and ' $B$ ' of figure 1(b). First of all, we study in ' $A$ ' $\left(\theta_{\mathrm{SH}}^{\mathrm{L}}=0.12\right)$ the dependence of the DW velocity-current relation as a function of $\theta_{\mathrm{SH}}^{\mathrm{U}}$.

For $\theta_{\mathrm{SH}}^{\mathrm{U}}=0$, the velocity-current relation is linear and the magnitude of the maximum velocity is $955 \mathrm{~m} \mathrm{~s}^{-1}$ for $j_{\mathrm{HM}} \sim$ $3.25 \times 10^{8} \mathrm{~A} \mathrm{~cm}^{-2}$ (figure 3(a) red squares). The key reason of such large velocity is a trade-off between the IEC torque, that tends to maintain the Néel DW configuration, and the SHE torque, that tends to rotate the DW inner magnetization towards a Bloch state. In other words, the large IEC allows for the injection of larger currents before reaching the saturation regime as well explained in [18]. As expected, in the absence of the edge roughness and/or other type of defects, no threshold current to move the DW is observed.

The effect of the $\theta_{\mathrm{SH}}^{\mathrm{U}}$ is summarized in figure 3(a). While for negative $\theta_{\mathrm{SH}}^{\mathrm{U}}(-0.06$ and -0.12 black circles, and olive up-triangles, respectively) the velocities grow proportionally, for positive values of $\theta_{\mathrm{SH}}^{\mathrm{U}}$ (blue down-triangles, $\theta_{\mathrm{SH}}^{\mathrm{U}}=0.06$ ), they decrease. In this case, the upper SHE acts as a 'brake' in slowing down the DW velocity. If $\theta_{\mathrm{SH}}^{\mathrm{U}}$ is exactly the same $(0.12$, not shown), the two SHE torques exactly balance each other and DW does not move. The non-linear behavior observed for $\theta_{\mathrm{SH}}^{\mathrm{U}}=-0.12$ at high current $\left(\left|j_{\mathrm{HM}}\right|>2.00 \times 10^{8} \mathrm{~A} \mathrm{~cm}^{-2}\right)$ is ascribed to the tilting of the DW inner magnetization towards 

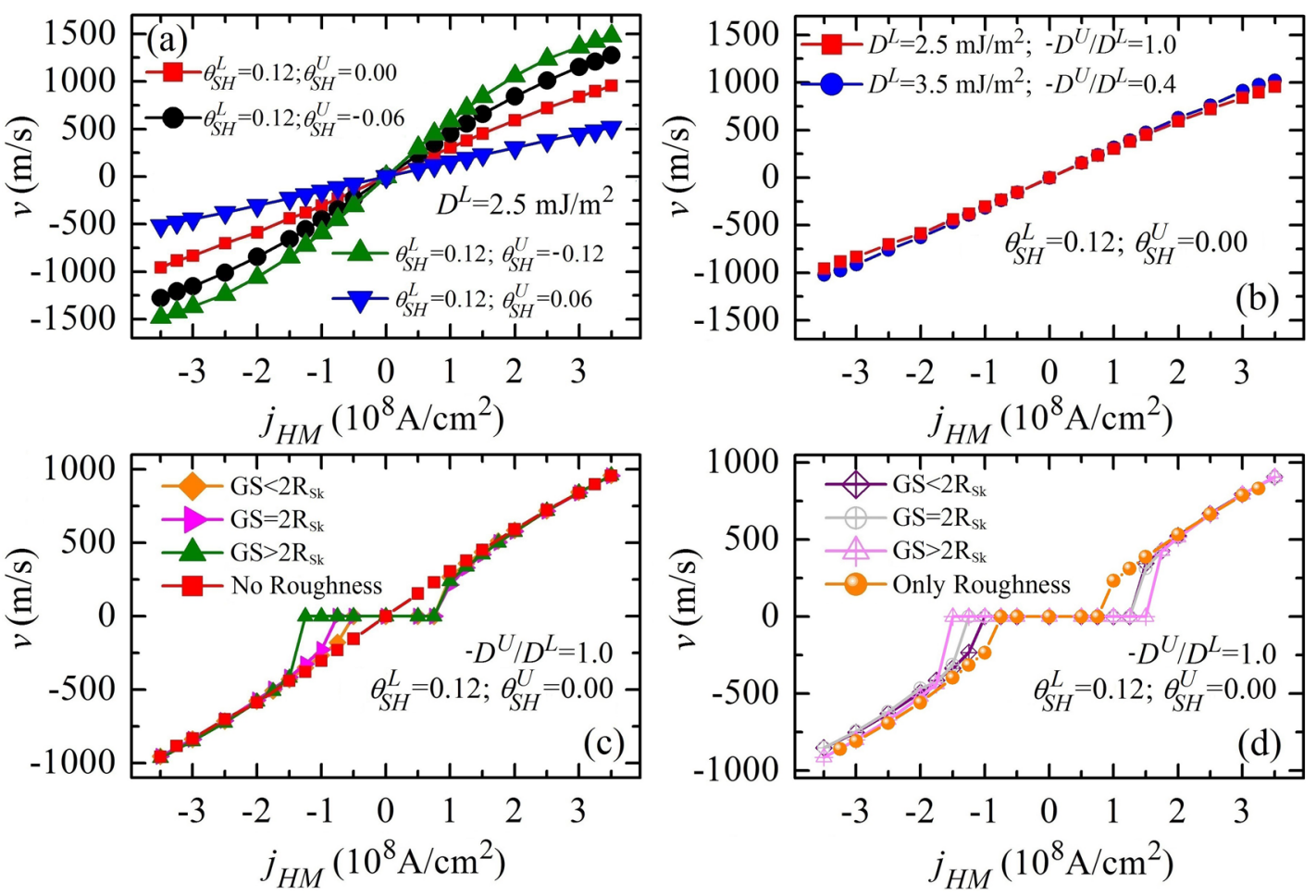

Figure 3. DW velocity-current relation in (a) working point 'A' of figure 1 (b) $\left(D^{\mathrm{L}}=2.5 \mathrm{~mJ} \mathrm{~m}{ }^{-2},-D^{\mathrm{U}} / D^{\mathrm{L}}=1.0\right)$, when $\theta_{\mathrm{SH}}^{\mathrm{L}}$ is fixed to 0.12 and $\theta_{\mathrm{SH}}^{\mathrm{U}}$ changes as indicated in the main panel, and (b) working point ' $\mathrm{B}$ ' of figure $1(\mathrm{~b})\left(D^{\mathrm{L}}=3.5 \mathrm{~mJ} \mathrm{~m}^{-2},-D^{\mathrm{U}} / D^{\mathrm{L}}=0.4\right)$ compared with 'A'. DW velocity-current relation in working point ' $A$ ' when the disordered anisotropy is introduced in a strip (c) without and (d) with edge roughness. The notations $\mathrm{GS}<2 \mathrm{R}_{\mathrm{Sk}}$, GS $=2 \mathrm{R}_{\mathrm{Sk}}$, and $\mathrm{GS}>2 \mathrm{R}_{\mathrm{Sk}}$ refer to an average grain size of $13 \mathrm{~nm}, 40 \mathrm{~nm}$ and $63 \mathrm{~nm}$, respectively.

the spin-polarization direction ( $y$-axis), leading to a reduction of the effective SHE torque acting on the DW.

This analysis leads to the second key result of this paper: the SHE angle of the upper HM should have a sign opposite to the lower HM one to enhance the velocity performance. To this aim, we can use the W (see appendix B). Figure 3(b) shows the velocity-current relation related to the working point ' $\mathrm{B}$ ', compared with the one of 'A'. No significant changes are observed. The DW velocity decreases while increasing the damping parameter, whereas it increases while reducing the thickness of the upper and lower FMs (not shown).

In order to provide a more realistic analysis, we also introduce a random variation of the perpendicular anisotropy constant $k_{\mathrm{u}}$ (disordered anisotropy) to simulate the presence of disorder (i.e. crystalline grains, defects, local variation of heavy metal and/or ferromagnet thickness can be accounted by these random variation of the PMA constant) [53, 54]. In particular, we introduce a $k_{\mathrm{u}}$ dispersion of $+10 \%$ around its mean value of $0.80 \mathrm{MJ} \mathrm{m}^{-3}$, and we create grains by means of the Voronoi tessellation algorithm. Three different patterns are studied, where the grain size has been determined in comparison with the skyrmion size. Specifically, the skyrmion diameter is $2 R_{\mathrm{Sk}}=40 \mathrm{~nm}$, therefore $\mathrm{GS}<2 R_{\mathrm{Sk}}, \mathrm{GS}=2 R_{\mathrm{Sk}}$, and GS $>2 R_{\text {Sk }}$ refer to grain sizes smaller, equal and larger than the skyrmion diameter: $\mathrm{GS}=13 \mathrm{~nm}, 40 \mathrm{~nm}$, and $63 \mathrm{~nm}$, respectively. Two different grain patterns have been considered for the upper and the lower FMs with a fixed mean grain size GS.
Figure 3(c) shows the results of the effect of the disordered anisotropy on DW motion in a strip with no edge roughness. The presence of grains induces an asymmetric DW pinning, which is higher for the largest GS considered here $\left(j_{\mathrm{HM}}=-1.25 \times 10^{8} \mathrm{~A} \mathrm{~cm}^{-2}\right)$. However, this asymmetric DW pinning is sample-to-sample dependent and this asymmetry is strongly reduced when the results are averaged over a large number of simulations for different grain patterns with the same characteristic size. Similar results are obtained when the disordered anisotropy is introduced in a rough strip (see figure 3(d)), where the combined effect of grains and edge roughness results in an increase of the depinning threshold current.

\subsection{SAF skyrmion motion}

Considering the working points ' $\mathrm{A}$ ' and ' $\mathrm{B}$ ', the current-driven skyrmion dynamics is also studied. The two skyrmions in the upper and the lower FMs move tied and do not exhibit either the skyrmion Hall effect (i.e. the component of the velocity ( $y$ axis) perpendicular to the electrical current direction ( $x$-axis) is zero [35]), or the transient breathing mode observed in [22]. Within a collective approach, the total skyrmion number $S$ is zero, as already described in [35]. The analysis of the effect of the spin-Hall angle leads to similar results as for the DW dynamics in term of influence of $\theta_{\mathrm{SH}}^{\mathrm{U}}$ (figures 4(a) and (b)) and of damping parameter and thicknesses of the upper and lower FMs (see appendix C). On the other hand, figure 4(c) shows 

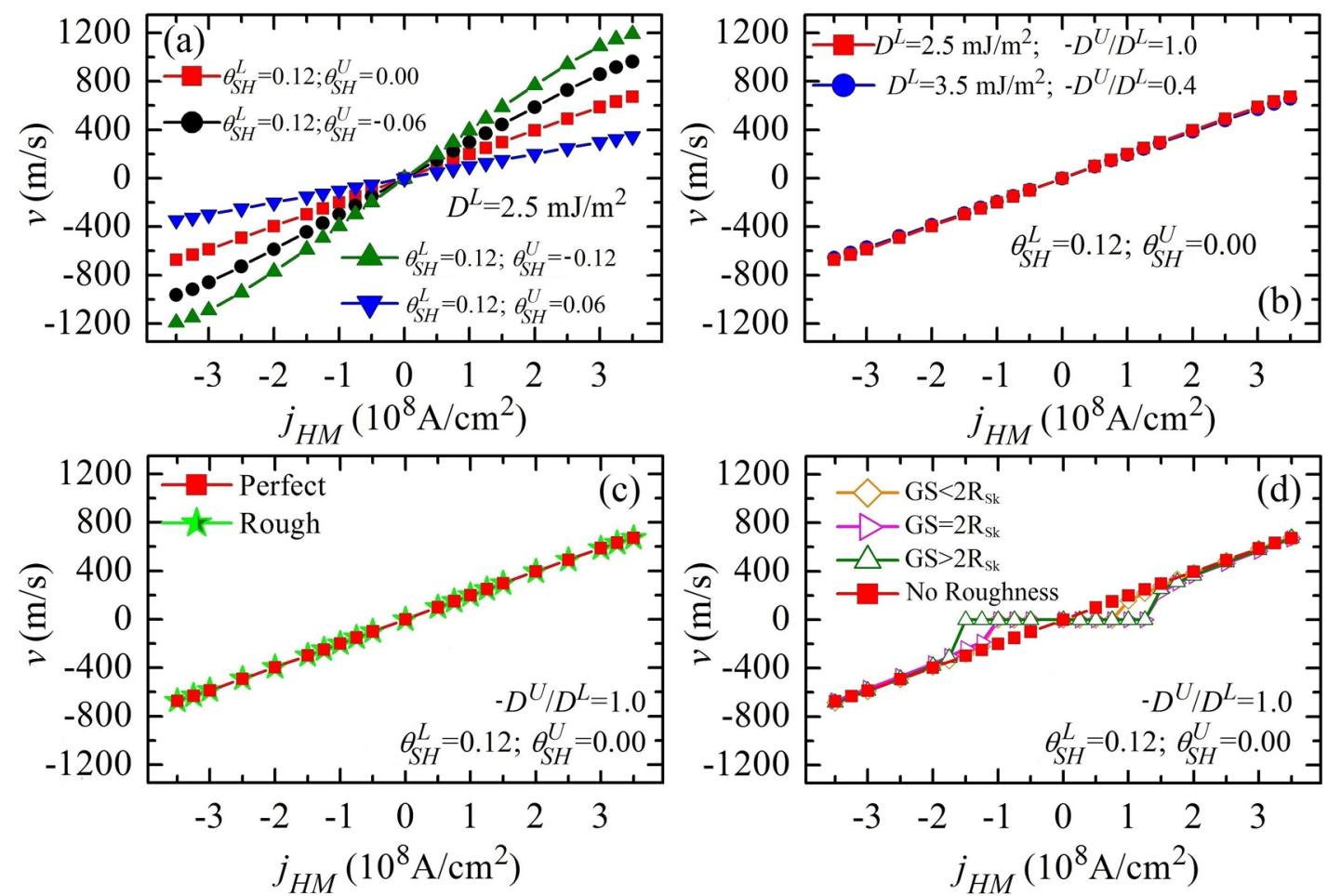

Figure 4. Skyrmion velocity-current relation in (a) working point ' $\mathrm{A}$ ' of figure 1 (b) $\left(D^{\mathrm{L}}=2.5 \mathrm{~mJ} \mathrm{~m}^{-2},-D^{\mathrm{U}} / D^{\mathrm{L}}=1.0\right)$, when $\theta_{\mathrm{SH}}^{\mathrm{L}}$ is fixed to 0.12 and $\theta_{\mathrm{SH}}^{\mathrm{U}}$ is changed as indicated in the main panel, (b) working point ' $\mathrm{B}$ ' of figure $1(\mathrm{~b})\left(D^{\mathrm{L}}=3.5 \mathrm{~mJ} \mathrm{~m}^{-2},-D^{\mathrm{U}} / D^{\mathrm{L}}=0.4\right)$ compared with 'A', and (c) working point ' $\mathrm{A}$ ', when $\theta_{\mathrm{SH}}^{\mathrm{L}}$ is fixed to 0.12 and $\theta_{\mathrm{SH}}^{\mathrm{U}}=0$, with (green stars) and without (red squares) edge roughness. (d) Skyrmion velocity-current relation in presence of disordered anisotropy compared with the case of racetrack without edge roughness (red squares). The notations $\mathrm{GS}<2 \mathrm{R}_{\mathrm{Sk}}, \mathrm{GS}=2 \mathrm{R}_{\mathrm{Sk}}$, and $\mathrm{GS}>2 \mathrm{R}_{\mathrm{Sk}}$ refer to an average grain size of $13 \mathrm{~nm}, 40 \mathrm{~nm}$, and $63 \mathrm{~nm}$, respectively.

that the skyrmion motion is not affected by the presence of the edge roughness as expected (see supplemental movie 7 to compare the skyrmion motion in the lower FM of a perfect and rough track for $j_{\mathrm{HM}}=1.50 \times 10^{8} \mathrm{~A} \mathrm{~cm}^{-2}$ ). This result makes SAF skyrmion racetrack more promising as compared to the one based on a single HM/FM bilayer, where the presence of the skyrmion Hall angle leads inevitably single skyrmion to interact with the edge roughness for high currents. Moreover, we analyze the effect of disordered anisotropy, as for DW motion. Also for the skyrmion, the disordered anisotropy gives rise to an asymmetric pinning that depends on the spatial distribution of the grains, and it is higher for the largest disordered anisotropy size (see figure $3(\mathrm{~d})$ ). This aspect is the key ingredient that originates a threshold current necessary for the skyrmion motion, in agreement with recent numerical achievements for single HM/FM bilayer [54]. In addition, those results point out that the disordered anisotropy is a fundamental feature to be taken into account when scaling the skyrmion diameter. Finally, the combined effect of disordered anisotropy and edge roughness does not significantly affect the velocity-current relations in the SAF heterostructure (not shown).

Some differences with recent works studying the skyrmion motion in SAF [35] and in antiferromagnetically-coupled structure [55] are briefly discussed below. Here, we have set the parameters of our computations after a benchmark with experimental data of the SAF DW dynamics. Zhang et al [35] deals with an IEC originated from spin-tunneling (the two ferromagnets are separated by an insulator). In [55], a real antiferromagnetic skyrmion motion is driven by the spin-transfer torque (STT) of the Zhang-Li form [56] due to an in-plane current. In ultrathin systems, where the ferromagnetic thickness is less than $1 \mathrm{~nm}$, it is very likely that the torque is mainly due to SHE (anti-damping torque of Slonczewski type) rather than STT. Indeed, due to the very thin thickness of the ferromagnets, most of the current flows through the heavy metals, so that the STT is usually negligible. In addition, the geometry of STT and SHE is profoundly different since the STT only cares about the magnetization gradient, while the SHE is uniquely sensitive to the chirality of the skyrmion (Néel, Bloch etc). A final remark is that the key drawback for antiferromagnetic solitons is their electrical detection.

\subsection{Comparison between the motion of DW and skyrmion in single HM/FM bilayer and in SAF}

We study, as reference, a racetrack made by a single HM/FM bilayer. The geometrical and physical parameters are: strip length along $y$-direction, width along $x$-direction and thickness $1000 \mathrm{~nm}, 100 \mathrm{~nm}$, and $1 \mathrm{~nm}$, respectively, $M_{\mathrm{s}}=1000 \mathrm{kA}$ $\mathrm{m}^{-1}, A=20 \mathrm{pJ} \mathrm{m}^{-1}, k_{\mathrm{u}}=0.8 \mathrm{MJ} \mathrm{m}^{-3}, \alpha_{G}=0.015, D=1.8$ $\mathrm{mJ} \mathrm{m}^{-2}, \theta_{\mathrm{SH}}=-0.33$ (same as Scenario B of [22]). Figure 5(a) shows the results of this study, highlighting that single DW is faster than single skyrmion for the whole current range, 

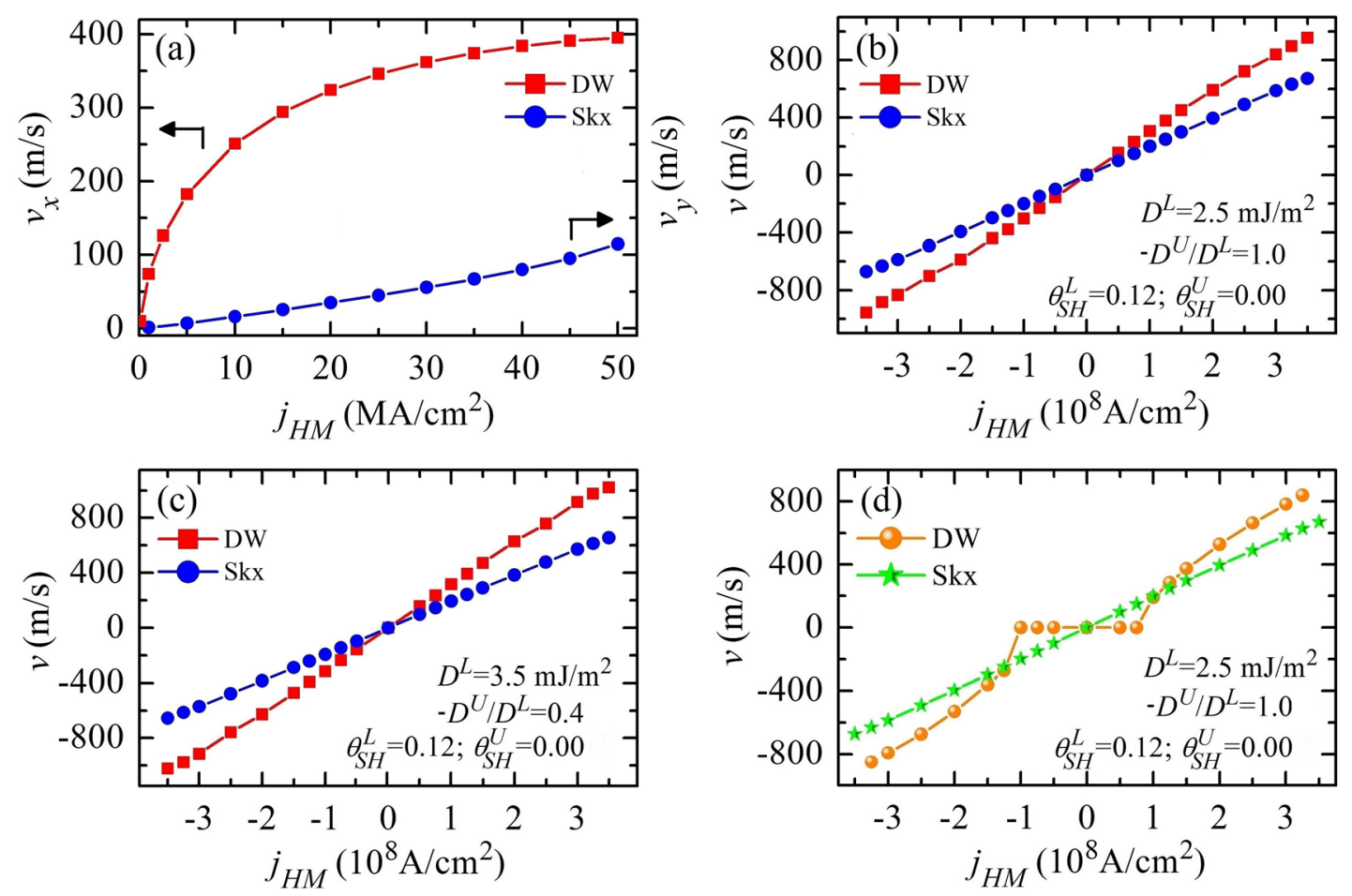

Figure 5. A comparison between the velocity-current relation of (a) single DW and single skyrmion in racetrack made by a HM/FM bilayer, (b) SAF DW and SAF skyrmion in working point ' $\mathrm{A}$ ' $\left(D^{\mathrm{L}}=2.5 \mathrm{~mJ} \mathrm{~m}{ }^{-2}, D^{\mathrm{U}} / D^{\mathrm{L}}=1.0\right)$, (c) SAF DW and SAF skyrmion in working point ' $\mathrm{B}$ ' $\left(D^{\mathrm{L}}=3.5 \mathrm{~mJ} \mathrm{~m}^{-2}, D^{\mathrm{U}} / D^{\mathrm{L}}=0.4\right)$, and (d), SAF DW and SAF skyrmion in working point 'A' including edge roughness. All the computations are performed for $\theta_{\mathrm{SH}}^{\mathrm{L}}=0.12$ and $\theta_{\mathrm{SH}}^{\mathrm{U}}=0.00$.
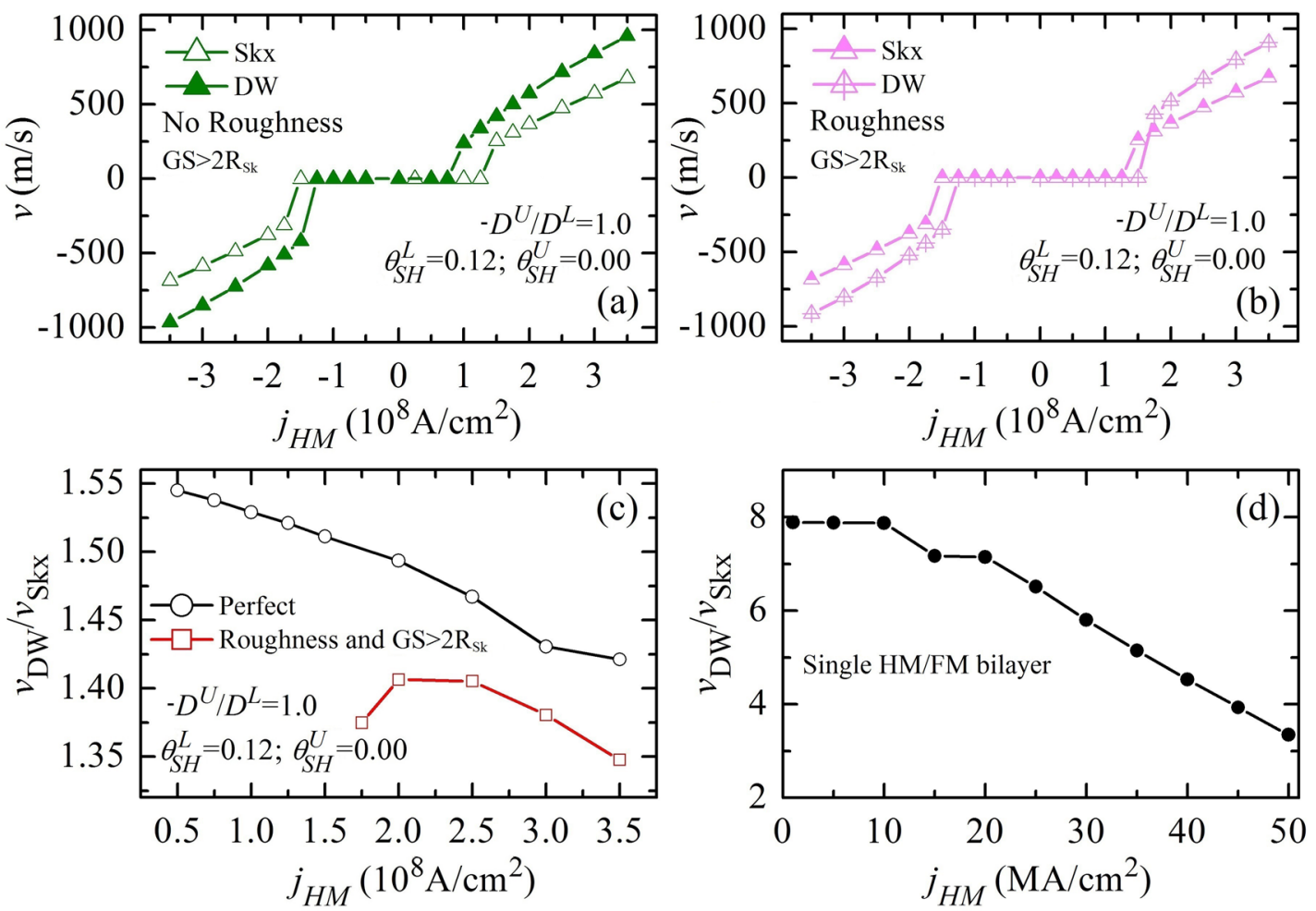

Figure 6. A comparison between the velocity-current relation of DW and skyrmion in a strip with disordered anisotropy with grains larger than the skyrmion diameter (GS $=63 \mathrm{~nm}>2 R_{\mathrm{Sk}}$ ), (a) without edge roughness, and (b) with edge roughness. (c), (d) Velocity ratio of DW and skyrmion in a SAF and single HM/FM bilayer racetrack, respectively. 
with a velocity difference of about $300 \mathrm{~m} \mathrm{~s}^{-1}$ for $\left|j_{\mathrm{HM}}\right|>30$ $\mathrm{MA} \mathrm{cm}^{-2}$. Similar results are also observed for other parameters.

The last part of the paper is focused on a comparison between SAF DW and SAF skyrmion motion. Let us consider the working points ' $\mathrm{A}$ ' $\left(D^{\mathrm{L}}=2.5 \mathrm{~mJ} \mathrm{~m}^{-2},-D^{\mathrm{U}} / D^{\mathrm{L}}=1.0\right.$, see figure $5(\mathrm{~b}))$ and ' $\mathrm{B}$ ' $\left(D^{\mathrm{L}}=3.5 \mathrm{~mJ} \mathrm{~m}^{-2},-D^{\mathrm{U}} / D^{\mathrm{L}}=0.4\right.$, see figure 5(c)), where only the SHE in the lower FM is taken into account. In a perfect strip, the DW velocity is larger than the skyrmion one.

Now, let us consider a strip with edge roughness. Figure 5(d) shows the results for the working point ' $A$ '. At low currents, when the DW remains pinned, skyrmion is faster (see supplemental movie 8 for the comparison of DW and skyrmion motion in the lower FM of a rough track, for $j_{\mathrm{HM}}=0.50 \times 10^{8}$ $\mathrm{A} \mathrm{cm}^{-2}$ ), whereas, for current values up to $2.00 \times 10^{8} \mathrm{~A} \mathrm{~cm}^{-2}$, their velocities are comparable. When $\left|j_{\mathrm{HM}}\right|>2.00 \times 10^{8}$ $\mathrm{A} \mathrm{cm}^{-2}$, the DW velocity is larger than the one of the skyrmion with a difference smaller than $200 \mathrm{~m} \mathrm{~s}^{-1}$ (see supplemental movie 9 for the comparison of DW and skyrmion motion in the lower FM of a rough track, for $j_{\mathrm{HM}}=3.25 \times 10^{8} \mathrm{~A} \mathrm{~cm}^{-2}$ ).

Finally, we compare SAF DW and SAF skyrmion motion in presence of disordered anisotropy $(\mathrm{GS}=13 \mathrm{~nm}$, $\mathrm{GS}=40 \mathrm{~nm}=2 \mathrm{R}_{\mathrm{Sk}}$, and $\mathrm{GS}=63 \mathrm{~nm}$ ). The disordered anisotropy introduces pinning for both DW and skyrmion dynamics, and the threshold current increases as the GS enlarges (compare figures 3(d) and 4(d)). Therefore, here we present the results for the worst case scenario only, i.e. when the grain size is larger than the skyrmion diameter (GS $\left.=63 \mathrm{~nm}>2 R_{\mathrm{Sk}}\right)$.

Figure 6(a) reports the comparison considering the disordered anisotropy only. The skyrmion exhibits a slightly larger depinning current and a smaller velocity than the DW one in the whole current range. This result demonstrates that the skyrmion motion is more sensitive to disordered anisotropy than DW. Figure 6(b) shows the combined effect of disordered anisotropy and edge roughness. DW and skyrmion exhibit the same depinning current and a comparable velocity up to $\left|j_{\mathrm{HM}}\right|=2.00 \times 10^{8} \mathrm{~A} \mathrm{~cm}^{-2}$ (see supplemental movie 10 for the comparison of DW and skyrmion motion in the lower FM of a rough track with disordered anisotropy, for $j_{\mathrm{HM}}=1.75 \times 10^{8}$ $\mathrm{A} \mathrm{cm}{ }^{-2}$ ). At larger currents, the DW is still faster than skyrmion (see supplemental movie 11 for the comparison of DW and skyrmion motion in the lower FM of a rough track with disordered anisotropy, for $j_{\mathrm{HM}}=3.00 \times 10^{8} \mathrm{~A} \mathrm{~cm}^{-2}$ ).

The results of the comparison are summarized in figures 6(c) and (d) where the ratio between DW and skyrmion velocity in a SAF racetrack (figure 6(c))) and in a single HM/FM bilayer (figure 6(d)) are shown. While in the latter, this ratio ranges between 3 to 8 , in SAF tracks, it is reduced in the range $1.35-1.55$, proving that the velocity of skyrmions in SAF racetracks is much closer to the one of DWs. This is the final quantitative important result of our work.

\section{Summary and conclusions}

In summary, we have studied a SAF racetrack based on both DW and skyrmion. The static analysis has pointed out that, while Néel DW can be stabilized in all the range of positive
IDMI ratio (including the case of zero upper IDMI), skyrmion can be obtained only if the upper IDMI is large enough. This result entails that, by considering state-of-the-art parameters, it is necessary to couple the upper ferromagnet to an upper $\mathrm{HM}$, which has to be characterized by an IDMI parameter sign opposite to the one of the lower HM.

For the motion driven by SHE, the presence of an upper $\mathrm{HM}$ introduces an additional degree of freedom for controlling both DW and skyrmion motion, allowing one to increase/ reduce the velocity if the two spin-Hall angles have different/ same sign. The comparison of DW and skyrmion in a track with edge roughness and disordered anisotropy has shown that (i) skyrmion velocity can approach the DW one in SAF racetrack, (ii) a SAF skyrmion, being not subject to the skyrmion Hall effect, is insensitive to the edge roughness, and (iii) the disordered anisotropy introduces pinning for both DW and skyrmion motion, and the threshold current increases as the grain size gets larger. In addition, racetrack based on SAF Skyrmion/DW can be more scalable in term of distance between two adjacent strips as compared with the standard racetracks based on single HM/FM bilayer. This is because the dipole interactions of the two opposite ferromagnetic layers partially cancel each other, thus inducing reduced disturbance as the FM strips becomes closer.

Finally, the use of SAF racetrack has a technical advantage over real antiferromagnetic racetrack: it is possible to achieve electrical detection of skyrmion by means of a magnetic tunnel junction (MTJ) built on top of the racetrack (a variation of the magnetic state below the MTJ is linked to a change in the tunneling magnetoresistive signal).

\section{Acknowledgments}

The authors acknowledge the executive programme of scientific and technological cooperation between Italy and China for the years 2016-2018 (code CN16GR09) title 'Nanoscale broadband spin-transfer-torque microwave detector' funded by Ministero degli Affari Esteri e della Cooperazione Internazionale. RT and MR also acknowledge Fondazione Carit-Projects-'Sistemi Phased-Array Ultrasonori', and 'Sensori Spintronici'. The work by EM was supported by project WALL, FP7-PEOPLE-2013-ITN 608031 from European Commission, project MAT2014-52477-C5-4-P from Spanish government, and project SA282U14 from Junta de Castilla y Leon. AM acknowledges support from the King Abdullah University of Science and Technology (KAUST). The authors acknowledge André Thiaville for very useful discussions.

\section{Appendix A. IDMI sign for the two heavy metals}

In order to stabilize the SAF configuration, the two heavy metals should be characterized by an opposite sign of the IDMI parameter. In fact, if we consider the SAF configuration for the UP and DOWN domains, and the same sign of the IDMI parameter for both heavy metals $\left(D^{\mathrm{U}}=D^{\mathrm{L}}\right)$, we obtain 


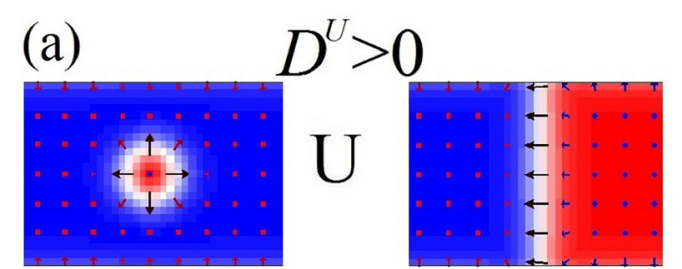

Ruthenium

Negative antiferromagnetic coupling

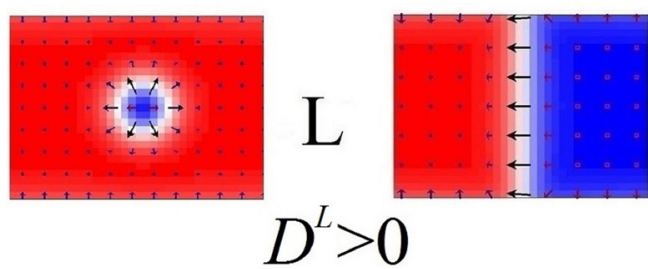

(b)

$D^{U}<0$

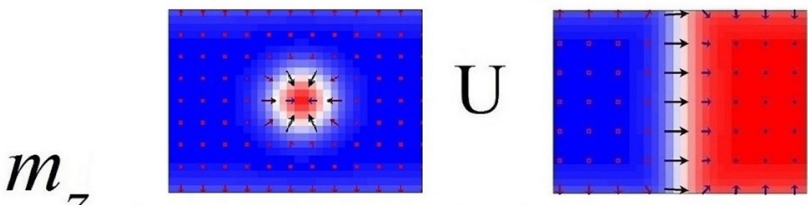

Ruthenium

$-1$

Negative antiferromagnetic coupling
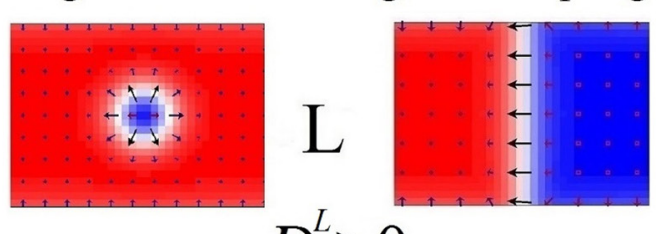

Figure A1. Spatial distribution of the magnetization in lower (L) and upper (U) ferromagnet for both skyrmion and DW, when (a) $D^{\mathrm{U}}=D^{\mathrm{L}}$, and (b) $D^{\mathrm{U}}=-D^{\mathrm{L}}$.

(a)

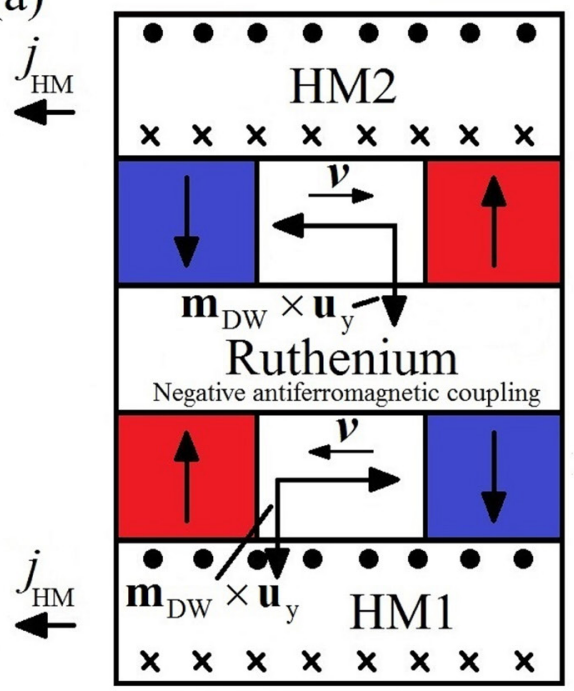

(b)

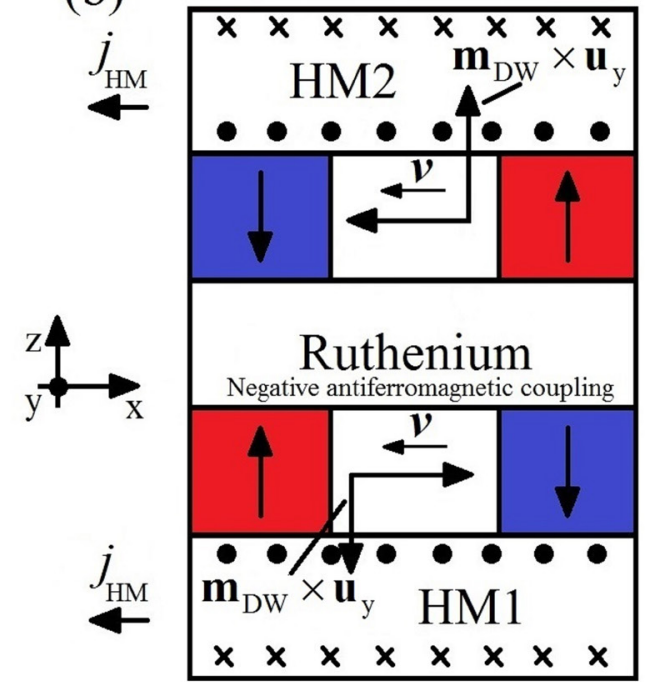

Figure A2. Sketch of SAF DW motion when the two heavy metals have (a) same and (b) opposite spin-Hall angle.
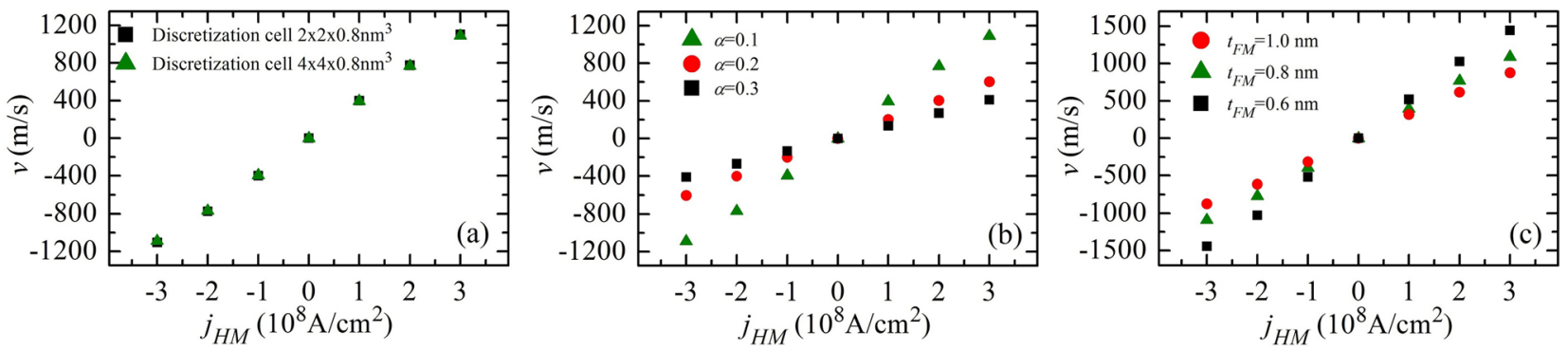

Figure A3. Velocity-current relation for SAF skyrmion in working point ' $\mathrm{A}$ ' $\left(D^{\mathrm{L}}=2.5 \mathrm{~mJ} \mathrm{~m}^{-2},-D^{\mathrm{U}} / D^{\mathrm{L}}=1.0\right)$, when $\theta_{\mathrm{SH}}^{\mathrm{L}}=0.12$ and $\theta_{\mathrm{SH}}^{\mathrm{U}}=-0.12$ when (a) the discretization cell size, (b) the damping parameter, and (c) the thickness of the upper and lower ferromagnets are changed.

an opposite chirality of both DW and skyrmion in the lower and upper ferromagnets (see figure A1(a)). On the other hand, if we consider an opposite sign of the IDMI parameter $\left(D^{\mathrm{U}}=-D^{\mathrm{L}}\right)$, we achieve the SAF configuration of both DW and skyrmion (see figure A1(b)), where both magnetic patterns are characterized by the same left-handed chirality. This is the reason why we have considered a negative IDMI ratio in figure 1(b) of the main text. We can conclude that, if, for instance, the lower HM is platinum, the upper one should be, for instance, tungsten. 


\section{Appendix B. Spin-Hall angle for the two heavy metals}

In order to enhance the velocity performance of SAF DW and skyrmion, the two heavy metals should be characterized by an opposite spin-Hall angle. In fact, if we consider that the two heavy metals have the same spin-Hall angle, i.e. the current flowing in the lower layer induces a positive spin-polarization (dots accumulating at the bottom interface), while the current flowing in the upper layer creates a negative spin-polarization (crosses accumulating at the bottom interface), the lower and upper DWs move in opposite directions (see figure A2(a)), leading to a reduction of the velocity (see figure 3(a) of the main text). On the other hand, if the two spin-Hall angles have an opposite sign, the two DWs move in the same direction (see figure A2(b)), yielding an increase of the velocity (see figure 3(a) of the main text). The same implications are valid for skyrmions. We can conclude that, if, for instance, the lower $\mathrm{HM}$ is platinum $\left(\theta_{\mathrm{SH}}^{\mathrm{L}} \approx 0.10[57,58]\right)$, the upper one should be tantalum $\left(\theta_{\mathrm{SH}}^{\mathrm{U}} \approx-0.15\right.$ [59]) or tungsten $\left(\theta_{\mathrm{SH}}^{\mathrm{U}} \approx-0.33\right.$ [60]). Since the combination platinum/tungsten is suitable for the IDMI sign as well, this could be a possible double heavy metal SAF configuration.

\section{Appendix C. Effect of discretization cell size, damping parameter and thickness of the ferromagnets}

We have performed further micromagnetic simulations for different cell size, damping or thickness of the ferromagnets, while maintaining all the other parameters fixed. In figure A3, we show the velocity-current relation for SAF skyrmion for the working point ' $\mathrm{A}$ ' $\left(D^{\mathrm{L}}=2.5 \mathrm{~mJ} \mathrm{~m}^{-2},-D^{\mathrm{U}} / D^{\mathrm{L}}=1.0\right)$, by considering $\theta_{\mathrm{SH}}^{\mathrm{L}}=0.12$ and $\theta_{\mathrm{SH}}^{\mathrm{U}}=-0.12$. The discretization cell size does not affect the results (see figure A3(a)). In figure A3(b), the increase of the damping yields a reduction of the velocity, in accordance with the Thiele's equation [22, 35] where the dissipative term is proportional to the damping parameter. In figure A3(c), the reduction of the thickness of the two ferromagnets leads to an increase of the velocity. This is mainly due to the increase of the spin-orbit torque term.

Qualitatively similar results are obtained for SAF DW.

\section{ORCID}

R Tomasello (1) https://orcid.org/0000-0002-9218-5633

V Puliafito (1) https://orcid.org/0000-0003-4438-538X

A Manchon (1) https://orcid.org/0000-0002-4768-293X

G Finocchio (D) https://orcid.org/0000-0002-1043-3876

\section{References}

[1] Åkerman J 2005 Toward a universal memory Science 308508

[2] Ikeda S, Miura K, Yamamoto H, Mizunuma K, Gan H D, Endo M, Kanai S, Hayakawa J, Matsukura F and Ohno H 2010 A perpendicular-anisotropy $\mathrm{CoFeB}-\mathrm{MgO}$ magnetic tunnel junction Nat. Mater. 9721
[3] Khalili Amiri P et al 2011 Switching current reduction using perpendicular anisotropy in $\mathrm{CoFeB}-\mathrm{MgO}$ magnetic tunnel junctions Appl. Phys. Lett. 98112507

[4] Tomasello R, Puliafito V, Azzerboni B and Finocchio G 2014 Switching properties in magnetic tunnel junctions with interfacial perpendicular anisotropy: micromagnetic study IEEE Trans. Magn. 507100305

[5] Kent A D and Worledge D C 2015 A new spin on magnetic memories Nat. Nanotechnol. 10187

[6] Parkin S S P, Hayashi M and Thomas L 2008 Magnetic domain-wall racetrack memory Science 320190

[7] Emori S, Bauer U, Ahn S-M, Martinez E and Beach G S D 2013 Current-driven dynamics of chiral ferromagnetic domain walls Nat. Mater. 12611

[8] Ryu K S, Thomas L, Yang S-H and Parkin S S P 2013 Chiral spin torque at magnetic domain walls Nat. Nanotechnol. 8527

[9] Parkin S and Yang S-H 2015 Memory on the racetrack Nat. Nanotechnol. 10195

[10] Zhang Y, Zhang C, Nan J, Zhang Z, Zhang X, Klein J-O, Ravelosona D, Sun G and Zhao W 2016 Perspectives of racetrack memory for large-capacity on-chip memory: from device to system IEEE Trans. Circuits Syst. I 63629

[11] Miron I M et al 2011 Fast current-induced domain-wall motion controlled by the Rashba effect Nat. Mater. 10419

[12] Fukami S, Suzuki T, Nakatani Y, Ishiwata N, Yamanouchi M, Ikeda S, Kasai N and Ohno H 2011 Current-induced domain wall motion in perpendicularly magnetized $\mathrm{CoFeB}$ nanowire Appl. Phys. Lett. 9882504

[13] Hirsch J E 1999 Spin Hall effect Phys. Rev. Lett. 831834

[14] Sinova J, Valenzuela S O, Wunderlich J, Back C H and Jungwirth T 2015 Spin Hall effects Rev. Mod. Phys. 871213

[15] Dzyaloshinsky I 1958 A thermodynamic theory of 'weak' ferromagnetism of antiferromagnetics J. Phys. Chem. Solids 4241

[16] Moriya T 1960 New Mechanism of anisotropic superexchange interaction_moriya Phys. Rev. Lett. 44

[17] Ryu K-S, Yang S-H, Thomas L and Parkin S S P 2014 Chiral spin torque arising from proximity-induced magnetization Nat. Commun. 53910

[18] Yang S-H, Ryu K-S and Parkin S 2015 Domain-wall velocities of up to $750 \mathrm{~m} \mathrm{~s}^{-5}$ driven by exchange-coupling torque in synthetic antiferromagnets Nat. Nanotechnol. 10221

[19] Fert A, Cros V and Sampaio J 2013 Skyrmions on the track Nat. Nanotechnol. 8152

[20] Iwasaki J, Mochizuki M and Nagaosa N 2013 Currentinduced skyrmion dynamics in constricted geometries Nat. Nanotechnol. 8742

[21] Sampaio J, Cros V, Rohart S, Thiaville A and Fert A 2013 Nucleation, stability and current-induced motion of isolated magnetic skyrmions in nanostructures Nat. Nanotechnol. 8839

[22] Tomasello R, Martinez E, Zivieri R, Torres L, Carpentieri M and Finocchio G 2014 A strategy for the design of skyrmion racetrack memories Sci. Rep. 46784

[23] Koshibae W, Kaneko Y, Iwasaki J, Kawasaki M, Tokura Y and Nagaosa N 2015 Memory functions of magnetic skyrmions Japan. J. Appl. Phys. 5453001

[24] Liu R H, Lim W L and Urazhdin S 2015 Dynamical skyrmion state in a spin current nano-oscillator with perpendicular magnetic anisotropy Phys. Rev. Lett. 114137201

[25] Zhou Y, Iacocca E, Awad A A, Dumas R K, Zhang F C, Braun H B and Akerman J 2015 Dynamically stabilized magnetic skyrmions Nat. Commun. 68193

[26] Carpentieri M, Tomasello R, Zivieri R and Finocchio G 2015 Topological, non-topological and instanton droplets driven by spin-transfer torque in materials with perpendicular 
magnetic anisotropy and Dzyaloshinskii-Moriya interaction Sci.Rep. 516184

[27] Finocchio G, Ricci M, Tomasello R, Giordano A, Lanuzza M, Puliafito V, Burrascano P, Azzerboni B and Carpentieri M 2015 Skyrmion based microwave detectors and harvesting Appl. Phys. Lett. 107262401

[28] Kang W, Huang Y, Zhang X, Zhou Y and Zhao W 2016 Skyrmion-electronics: an overview and outlook Proc. IEEE 1042040

[29] Jiang W et al 2015 Blowing magnetic skyrmion bubbles Science 349283

[30] Woo $\mathrm{S}$ et al 2016 Observation of room-temperature magnetic skyrmions and their current-driven dynamics in ultrathin metallic ferromagnets Nat. Mater. 15501

[31] Finocchio G, Büttner F, Tomasello R, Carpentieri M and Kläui M 2016 Magnetic skyrmions: from fundamental to applications J. Phys. D: Appl. Phys. 49423001

[32] Tomasello R, Ricci M, Burrascano P, Puliafito V, Carpentieri M and Finocchio G 2017 Electrical detection of single magnetic skyrmion at room temperature $A I P A d v$. 756022

[33] Jiang W et al 2016 Direct observation of the skyrmion Hall effect Nat. Phys. 13162

[34] Litzius K et al 2016 Skyrmion Hall effect revealed by direct time-resolved x-ray microscopy Nat. Phys. 13170

[35] Zhang X, Zhou Y and Ezawa M 2016 Magnetic bilayerskyrmions without skyrmion Hall effect Nat. Commun. 710293

[36] Slonczewski J C 1989 Conductance and exchange coupling of two ferromagnets separated by a tunneling barrier Phys. Rev. B 396995

[37] Faure-Vincent J, Bellouard C T C, Popova E, Hehn M, Montaigne F and Schuhl A 2002 Interlayer magnetic coupling interactions of two ferromagnetic layers by spin polarized tunneling Phys. Rev. Lett. 89107206

[38] Parkin S S P and Mauri D 1991 Spin engineering: direct determination of the Ruderman-Kittel-Kasuya-Yosida far-field range function in ruthenium $P$ hys. Rev. B 447131

[39] Giordano A, Carpentieri M, Laudani A, Gubbiotti G, Azzerboni B and Finocchio G 2014 Spin-Hall nanooscillator: a micromagnetic study Appl. Phys. Lett. 10542412

[40] Siracusano G, Tomasello R, Giordano A, Puliafito V, Azzerboni B, Ozatay O, Carpentieri M and Finocchio G 2016 Magnetic radial vortex stabilization and efficient manipulation driven by the Dzyaloshinskii-Moriya interaction and spin-transfer torque Phys. Rev. Lett. 11787204

[41] Siracusano G and La Corte A 2014 A comparison between advanced time-frequency analyses of non-stationary magnetization dynamics in spin-torque oscillators Physica B 43566

[42] Grytsyuk S, Belabbes A, Haney P M, Lee H-W, Lee K-J, Stiles M D, Schwingenschlögl U and Manchon A 2016 $k$-asymmetric spin splitting at the interface between transition metal ferromagnets and heavy metals Phys. Rev. B 93174421

[43] Rohart S and Thiaville A 2013 Skyrmion confinement in ultrathin film nanostructures in the presence of
Dzyaloshinskii-Moriya interaction Phys. Rev.

B 88184422

[44] Tomasello R, Carpentieri M and Finocchio G 2014 Influence of the Dzyaloshinskii-Moriya interaction on the spin-torque diode effect J. Appl. Phys. 115 17C730

[45] Schutte C and Garst M 2014 Magnon-skyrmion scattering in chiral magnets Phys. Rev. B 9094423

[46] Belmeguenai M, Adam J P, Roussigné Y, Eimer S, Devolder T, Von Kim J, Cherif S M, Stashkevich A and Thiaville A 2015 Interfacial Dzyaloshinskii-Moriya interaction in perpendicularly magnetized $\mathrm{Pt} / \mathrm{Co} / \mathrm{AlO}_{x}$ ultrathin films measured by Brillouin light spectroscopy Phys. Rev. B 91180405

[47] Cubukcu M, Sampaio J, Bouzehouane K, Apalkov D, Khvalkovskiy A V, Cros V and Reyren N 2016 Dzyaloshinskii-Moriya anisotropy in nanomagnets with in-plane magnetization Phys. Rev. B 93020401

[48] Boulle O et al 2016 Room temperature chiral magnetic skyrmion in ultrathin magnetic nanostructures Nat. Nanotechnol. 11449

[49] Nakatani Y, Thiaville A and Miltat J 2003 Faster magnetic walls in rough wires Nat. Mater. 2521

[50] Martinez E, Lopez-Diaz L, Torres L, Tristan C and Alejos O 2007 Thermal effects in domain wall motion: micromagnetic simulations and analytical model Phys. Rev. B 75174409

[51] Nguyen M H, Ralph D C and Buhrman R A 2016 Spin torque study of the spin Hall conductivity and spin diffusion length in platinum thin films with varying resistivity Phys. Rev. Lett. 116126601

[52] Boulle O, Rohart S, Buda-Prejbeanu L D, Jué E, Miron I M, Pizzini S, Vogel J, Gaudin G and Thiaville A 2013 Domain wall tilting in the presence of the Dzyaloshinskii-Moriya interaction in out-of-plane magnetized magnetic nanotracks Phys. Rev. Lett. 111217203

[53] Voto M, Lopez-Diaz L and Torres L 2016 Effects of grain size and disorder on domain wall propagation in $\mathrm{CoFeB}$ thin films J. Phys. D: Appl. Phys. 49185001

[54] Kim J-V and Yoo M-W 2017 Current-driven skyrmion dynamics in disordered films Appl. Phys. Lett. 110132404

[55] Barker J and Tretiakov O A 2016 Static and dynamical properties of antiferromagnetic skyrmions in the presence of applied current and temperature Phys. Rev. Lett. 116147203

[56] Zhang S and Li Z 2004 Roles of nonequilibrium conduction electrons on the magnetization dynamics of ferromagnets Phys. Rev. Lett. 93127204

[57] Liu L, Moriyama T, Ralph D C and Buhrman R A 2011 Spintorque ferromagnetic resonance induced by the spin Hall effect Phys. Rev. Lett. 10636601

[58] Liu L, Lee O J, Gudmundsen T J, Ralph D C and Buhrman R A 2012 Current-induced switching of perpendicularly magnetized magnetic layers using spin torque from the spin hall effect Phys. Rev. Lett. 109096602

[59] Liu L, Pai C-F, Li Y, Tseng H W, Ralph D C and Buhrman R A 2012 Spin-torque switching with the giant spin Hall effect of tantalum Science 336555

[60] Pai C F, Liu L, Li Y, Tseng H W, Ralph D C and Buhrman R A 2012 Spin transfer torque devices utilizing the giant spin Hall effect of tungsten Appl. Phys. Lett. 101122404 